\title{
Conditioned flavor preferences as a function of deprivation level: Preferences or aversions?
}

\author{
DAVID H. CAMPBELL, ELIZABETH DEUTSCH CAPALDI, and DAVID E. MYERS \\ Purdue University, West Lafayette, Indiana
}

\begin{abstract}
Rats tend to prefer flavors previously consumed under low deprivation to flavors previously consumed under high deprivation (Capaldi \& Myers, 1982). We attempted to distinguish among possible associative explanations by determining whether this conditioning phenomenon was based upon conditioned preferences, conditioned aversions, or both. We compared preference for flavors presented exclusively under either high or low deprivation with preference for a neutral flavor. In Experiments 1A and 1B the neutral flavor was one that had been randomly paired with both high and low deprivation, whereas in Experiments 2 and 3 the neutral flavors had not been associated with either high or low deprivation. Our results strongly suggest that this conditioning phenomenon is based upon an actual increase in preference for the flavor consumed under low deprivation rather than on any form of aversion conditioning.
\end{abstract}

Rats tend to prefer a flavor originally consumed under low deprivation to one originally consumed under high deprivation, so long as the flavors are not closely associated with daily feedings (see Capaldi \& Myers, 1982; Capaldi, Myers, Campbell, \& Sheffer, 1983). These data appear to be contrary to the traditional assumption that the incentive value of food reward is an increasing function of deprivation level (e.g., Kurtz \& Jarka, 1968). The purpose of the present investigation was to identify the mechanism underlying this conditioning phenomenon.

According to some hypotheses, the flavor consumed under high deprivation becomes associated with something aversive; thus, the apparent preference for the lowdeprivation flavor is actually due to a decreased evaluation of the high-deprivation flavor. For example, Capaldi and Myers (1982) proposed that the small samples of sweet flavor solution presented during training might elicit digestive responses that were unsatisfied due to insufficient caloric intake. They hypothesized that the unsatisfied digestive responses might be greater and more aversive under high deprivation than under low deprivation. Consequently, preference for the high-deprivation flavor would decrease more than preference for the lowdeprivation flavor as a result of pairing with more aversive postingestive consequences. Similarly, the flavor consumed under high deprivation might become associated with aversive properties of deprivation per se (see, e.g., Davidson-Codjoe \& Holman, 1982). Such explanations assume that the effective unconditioned stimulus (US) is aversive, and that this phenomenon is actually one of conditioned aversions rather than conditioned preferences.

On the other hand, it is possible that the conditioned preference observed in this procedure is actually due to the rats' enhanced evaluation for the low-deprivation

This research was supported in part by Research Grant RO1 MH39453 from the Department of Mental Health. Send requests for reprints to David H. Campbell, Department of Psychology, The College of Wooster, Wooster, OH 44691 . flavor. Capaldi et al. (1983) suggested that the flavored solutions might taste better when consumed under low deprivation than when consumed under high deprivation. Similarly, Davidson-Codjoe and Holman's (1982) hypothesis that flavors become associated with aspects of the deprivation condition may be interpreted to mean that the flavor consumed under low deprivation becomes associated with pleasant properties of low deprivation (although their hypothesis is also compatible with the idea that high deprivation is aversive, and so aversion conditioning is involved)

One reason it is difficult to choose an interpretation of these data is that we have little information on whether preferences, aversions, or both are being conditioned. In all previous experiments, the measure of preference between flavors previously associated with high and low deprivation has been a relative one; it has not been known whether the change in relative preference was due to an increased preference for the flavor previously associated with low deprivation, a decreased preference for the flavor associated with high deprivation, or a combination of both. Our aim in the present research was to determine whether the value of the high-deprivation flavor decreased as a result of conditioning, and/or whether the value of the low-deprivation flavor increased as a result of conditioning. To determine the direction in which preference shifted as a result of this conditioning procedure, we compared the preferences of experimental subjects, who had flavors paired with deprivation conditions, with those of control subjects, who did not have flavors previously paired with a specific deprivation.

\section{EXPERIMENTS 1A AND 1B}

In the first two experiments we compared preference for flavors uniquely associated with high and low deprivation with flavors randomly associated with both high and low deprivation. We wanted to compare the prefer- 
ence for a flavor paired with each deprivation condition against the preference for a neutral flavor. Since there is no single deprivation level that is neutral, we attempted to establish the relative neutrality of a flavor by pairing it with both high- and low-deprivation conditions. If the critical US is exclusively either high or low deprivation, then a randomly paired flavor is associated equally with the presence and the absence of the US (somewhat like Rescorla's [1967] truly random control procedure). If critical USs are both low- and high-deprivation presentations, then the randomly paired flavor is associated at least equally often with both and does not portend either level of deprivation. To the degree that pairing with high deprivation decreases preference for the high-deprivation flavor by aversive conditioning, a randomly paired flavor might be preferred to this high-deprivation flavor. If pairing with low deprivation actually increases preference for the lowdeprivation flavor, the low-deprivation flavor might be preferred to the randomly paired flavor. Note that this approach to creating a neutral stimulus is based upon a contingency view of classical conditioning.

Subjects in Group HL (high and low) received one flavor on 10 high-deprivation days and the other flavor on 10 low-deprivation days. This represented the standard conditioning procedure employed in previous experiments.

Groups HR (high and random) and LR (low and random) were the critical groups. Subjects in Group HR received one flavor on 10 high-deprivation days and the other flavor on 5 low- and 5 high-deprivation days. If the flavor paired with both low and high deprivation (the randomly paired flavor) was neutral, a preference for this flavor over the flavor associated with high deprivation in this group should indicate a conditioned aversion to the high-deprivation flavor. Subjects in Group LR received one flavor on $10 \mathrm{low}$-deprivation days and the other flavor on 5 high- and 5 low-deprivation days. Preference for the flavor associated with low deprivation over the randomly paired flavor in this group should indicate conditioned preference for the low-deprivation flavor. If this preference-conditioning phenomenon were based exclusively on an aversion to the high-deprivation flavor and the randomly paired flavors were neutral, then conditioned preference in Groups HL and HR should be comparable and there should be no conditioned preference in Group LR. On the other hand, if this preference were based exclusively on increased liking for the lowdeprivation flavor and the randomly paired flavors were neutral, then there should be comparable conditioned preference in Groups HL and LR and no conditioned preference in Group HR. If there were both an aversion conditioned to the high-deprivation flavor and a preference conditioned to the low-deprivation flavor, then the conditioned preference in Group HL should be greater than that in Group HR or Group LR.

In Experiment 1B we included two additional groups ( $H$ and $L$ ) to assess the effects of the number of high- or low-deprivation pairings alone on conditioned flavor preference. If conditioning of aversions or preferences were asymptotic after five pairings, there should be no difference in preference between cues paired 5 times and cues paired 10 times with a particular deprivation state. To interpret the results obtained by comparing Groups HL, HR, and LR, it is important to know the effect of number of pairings. Group HL received 10 pairings of one flavor with high deprivation and 10 pairings of another flavor with low deprivation. Group HR received 10 pairings of one flavor with high deprivation and a second flavor that was paired with high deprivation 5 times and low deprivation 5 times. If Group HR preferred the flavor paired with both low and high deprivation to the flavor paired only with high deprivation, this might be because of the low-deprivation pairings or it might be because a flavor paired with high deprivation only 5 times is preferred to a flavor paired with high deprivation 10 times. In Experiment 1B, Group $H$ received one flavor paired with high deprivation 5 times and the other flavor paired with high deprivation 10 times. Preference in this group would give a measure of the effects of 5 versus 10 pairings with high deprivation in producing flavor preferences. Group $L$ in Experiment 1B received 10 pairings of Flavor 1 with low deprivation and 5 pairings of Flavor 2 with low deprivation. Preference in this group would give a measure of the effects of 5 versus 10 pairings with low deprivation in producing flavor preferences. This information would be important for interpreting the results for Group LR in Experiment 1A. Group LR received 10 pairings of Flavor 1 with low deprivation, 5 pairings of Flavor 2 with low deprivation, and 5 pairings of Flavor 2 with high deprivation. Thus, if Flavor 1 were preferred, it might be because high-deprivation pairings produce an aversion, or because a flavor paired with low deprivation 5 times is less preferred than a flavor paired with low deprivation 10 times, or both. Group L would show the extent to which differing numbers of pairings with low deprivation contributed to the preference obtained in Group LR.

\section{Method}

Subjects. The subjects in Experiment 1A were 36 male albino rats from the Holtzman Company, Madison, Wisconsin, 196 days old at the beginning of the experiment. They had had prior experience in a straight-alley instrumental learning experiment. The subjects in Experiment $1 \mathrm{~B}$ were 60 rats from the same source and were 168-189 days old at the beginning of the experiment. They had been used previously in shuttle-avoidance or straight-alley instrumental learning experiments. The subjects were divided into groups so as to balance as closely as possible for their previous group assignments. In Experiment 1A the subjects were divided into three main groups with counterbalanced subgroups of 6 subjects each. In Experiment 1B there were five main groups with counterbalanced subgroups of 10 subjects each.

Materials. Flavored solutions were presented in 50-ml Nalgene centrifuge tubes with rubber stoppers and metal spouts. Flavor cues consisted of $1 \%$ cinnamon (C) or wintergreen (W) (2\% imitation cinnamon or wintergreen flavoring mixed with $100 \%$ ethanol) dissolved in $.15 \%$ sodium saccharin solution.

Procedure. The rats were housed individually, and all training and testing took place in the rats' home cages. Water was available ad lib throughout the experiment in a 350-ml brown bottle mounted on the right-hand side of each cage front. Food was removed from 
each cage on the day preceding Day 1 of the experiment. Beginning on Day 1 , each rat received $35 \mathrm{~g}$ of food late in the afternoon on even-numbered days but no food on odd-numbered days. This alternating feeding schedule continued throughout training and testing.

Following adaptation to the deprivation schedule (11 days in Experiment $1 \mathrm{~A}$ and 32 days in Experiment $1 B$ ), all subjects were exposed to flavors under high and/or low deprivations. The flavors were presented at 9:00 a.m., following feeding on low- and highdeprivation days by 16 and $40 \mathrm{~h}$, respectively, in Experiment 1A, and by 18 and $42 \mathrm{~h}$, respectively, in Experiment 1B. The absolute hours of deprivation differed slightly from study to study because of the schedule requirements of the experimenters. There was always a 24-h difference between low deprivation and high deprivation. Experiments in our laboratory have shown that such differences in deprivation do not affect the phenomenon under investigation. To determine if stronger preferences could be conditioned if larger samples of the flavor solution were presented, 5-ml flavor samples were given in Experiment $1 \mathrm{~A}$ and 10-ml samples were given in Experiment 1B.

The training phase lasted for 30 days -15 high-deprivation days (odd-numbered days) and 15 low-deprivation days (even-numbered days). Group HL received one flavor on 10 high-deprivation mornings and the other flavor on 10 low-deprivation mornings. Group HR received one flavor on $\mathbf{1 0}$ high-deprivation mornings and the second flavor on 5 different high-deprivation mornings and 5 lowdeprivation mornings. Group LR received one flavor on 10 lowdeprivation mornings and the second flavor on 5 different lowdeprivation mornings and 5 high-deprivation mornings. Group $\mathrm{H}$ received one flavor on $\mathbf{1 0}$ high-deprivation mornings and the other flavor on 5 different high-deprivation mornings. Group $L$ received one flavor on $\mathbf{1 0}$ low-deprivation mornings and the other flavor on 5 different low-deprivation mornings. High-deprivation flavors for Groups HL, HR, and $H$ were presented on Days $1,5,7$, $11,13,15,19,23,25$, and 29 . Low-deprivation flavors for Groups $H L, L R$, and $L$ were presented on Days $4,6,10,12$, $18,20,22,24$, and 26 . High-deprivation pairings for the flavor paired with both high and low deprivation for Groups HR and LR and for the flavor paired with high deprivation five times for Group $H$ were presented on Days 3, 9, 17, 21, and 27. Lowdeprivation pairings for the flavor paired with both high and low deprivation for Group $\mathrm{HR}$ and $\mathrm{LR}$ and for the flavor paired with low deprivation five times for Group $L$ were presented on Days $6,10,12,22$, and 26.

Following training, preference for the flavors was tested. The same alternating deprivation schedule continued throughout testing, and subjects were tested at the same time of day as they had been in training. In Experiment $1 \mathrm{~A}$, on each of the first 4 days of testing, the subjects were given $\mathbf{2 0}$ min exposure to a single tube containing $\mathbf{4 5} \mathrm{ml}$ of flavored solution. For half of the subjects in each group, the onder of flavor presentation was Flavor 1, Flavor 2, Flavor 2, Flavor 1; for the other half it was Flavor 2, Flavor 1, Flavor 1, Flavor 2. When we found no conditioned preference, we introduced a more sensitive simultaneous choice test on the subsequent 4 test days. This simultaneous choice procedure was used on all 8 test days in Experiment 1B. Two tubes containing $40 \mathrm{ml}$ of $W$ and $C$ solution were presented simultaneously. The first tube sampled was withdrawn; when the second tube was sampled, it was withdrawn, and both tubes were then reinserted. All tubes were removed after $10 \mathrm{~min}$ and the amount of residual solution was recorded. For half of the subjects in each subgroup, $C$ was on the left and $W$ was on the right on Days 1, 4 (Experiment 1B only), 5 , and 8 , and the reverse was true on the other 4 days; for the other half of each subgroup, the flavor positions were reversed. A subject from Group $H$ died during Experiment 1B, and its data were discarded.

\section{Results}

In these experiments our primary interest was in the strength of conditioned flavor preferences across groups. We have chosen (arbitrarily) to evaluate conditioned preferences for $W$. Preference for $W$ is defined as milliliters of W consumed divided by total milliliters consumed of $W$ and $C$. Evaluation of the conditioned preference in terms of the proportion of $W$ consumed is completely arbitrary; the differences in proportion scores among subgroups (the critical comparison) are exactly the same whether proportion of $W$ or proportion of $C$ is compared.

The proportions of $W$ consumed by each subgroup in Experiments 1A and 1B are shown in Table 1. For Experiment $1 \mathrm{~A}$, only the results for two-bottle testing are shown. In the left-hand column of this table, the proportion of $W$ consumed is shown for the subgroups for which W was associated with the low-deprivation level (the flavor paired 10 times with low deprivation for Groups HL, LR, and L; the flavor paired with both low and high deprivation for Group HR; and the flavor paired with high deprivation 5 times for Group $\mathbf{H}$ ). In the righthand column of Table 1 the proportion of $W$ consumed is given for the subgroups in which $W$ was paired with the high-deprivation conditions (the flavor paired 10 times with high deprivation for Groups HL, HR, and H; the flavor paired with low and high deprivation for Group LR; and the flavor paired 5 times with low deprivation for Group L). Conditioned preference for any group may be assessed by comparing the difference in the proportion of $W$ consumed across the low- and high-deprivation subgroups in which W was associated with opposite deprivation conditions. Such a between-subgroups measure of conditioned preference eliminates the effects of any extraneous factors associated with group treatments that may interact with preference for a particular flavor. Any such factors that affect preference are canceled out with a between-groups measure of conditioned preference, because they affect both subgroups comparably.

As can be seen by comparing the proportions in the two columns in Table 1, for every group, $\mathrm{W}$ was preferred

Table 1

Mean Preferences for Wintergreen in Terms of Proportions Consumed in Experiments 1A and 1B

\begin{tabular}{ccc}
\hline & \multicolumn{2}{c}{$\begin{array}{c}\text { Deprivation Level } \\
\text { Paired with Wintergreen }\end{array}$} \\
\cline { 2 - 3 } Group & Low & High \\
\hline HL & Experiment 1A \\
HR & .61 & .33 \\
LR & .59 & .35 \\
& .52 & .29 \\
HL & Experiment 1B & \\
HR & .70 & .19 \\
H & .53 & .28 \\
LR & .42 & .40 \\
L & .59 & .34 \\
\end{tabular}


more when it was paired with low deprivation than when it was paired with high deprivation. Analysis of variance on the proportion of $W$ consumed in these tests for $\mathrm{Ex}$ periment $1 A$ confirmed that $W$ was preferred more when it had been associated with the low-deprivation condition $[F(1,30)=15.12, p<.001]$ and that the size of the conditioned preference did not differ across groups $(F<1)$. This analysis involved group (HL vs. HR vs. LR) and subgroup ( $W$ as the low- or high-deprivation flavor) as factors. In other words, the differences in proportion scores among subgroups were comparable across the three groups.

Analysis of variance on proportion scores from Experiment $1 \mathrm{~B}$ also revealed that overall there was a greater preference for $W$ when $W$ was the low-deprivation flavor $[F(1,49)=47.54, p<.001]$, but in this case preference did differ by group $[F(4,49)=6.01, p<.001]$.

Further analysis of individual group differences in Experiment 1B, employing the overall between-subjects error term, revealed that a significantly greater proportion of $W$ was consumed in the low-deprivation subgroups than in the high-deprivation subgroups for Groups HL, HR, and LR [smallest $F(1,49)=11.17, p<.01$ ], but this difference was not significant for Group $H(F<1)$ or Group $\mathrm{L}[F(1,49)=2.50, p>.05]$. This means that there was a significant conditioned preference only in Groups HL, HR, and LR.

The group $\times$ subgroup interaction was broken down to compare the strength of this preference across pairs of groups, using the error term from the overall interaction. Conditioned preference (indicated by the difference between subgroup proportion scores) was greater in Group HL than in either Group HR $[F(1,49)=6.16$, $p<.05]$ or Group LR $[F(1,49)=5.59, p<.05]$, whereas preference between Groups HR and LR did not differ $(F<1)$. Group HR showed a significantly greater preference for the cue associated with low deprivation than did Group $H[F(1,49)=4.44, p<.05]$. Note that these two groups were treated identically except for the five additional pairings of the low-deprivation flavor cue with low deprivation in Group HR. The strength of preference did not, however, differ significantly between Groups LR and $L[F(1,49)=1.84, p>.05]$, which were treated identically except for the five additional pairings of the high-deprivation flavor cue with high deprivation in Group LR.

Since the analysis of absolute consumption in both experiments revealed the same pattern of results, the findings will not be discussed independently. Also, since the results were the same in both experiments on both highand low-deprivation test days, the data are not presented separately by deprivation condition in testing.

\section{Discussion}

These experiments confirm the general observation of preference for a flavor paired with low deprivation over a flavor paired with high deprivation. Our new observations are that a flavor paired exclusively with low depri- vation was preferred to a flavor randomly paired with low and high deprivation (Group LR), and that a flavor randomly paired with low and high deprivation was preferred to a flavor paired exclusively with high deprivation (Group HR). If the randomly paired flavors are actually neutral (which, we will see, there is reason to question), these observations suggest that the training procedure used in Experiments 1A and $1 \mathrm{~B}$ results in both conditioned preference for the low-deprivation flavor and conditioned aversion to the high-deprivation flavor. Compatible with this conclusion, in Experiment 1B relative preference for the low-deprivation flavor (the proportion score for the low-deprivation subgroup minus the proportion score for the high-deprivation subgroup) in Group HL was approximately double what it was in Group HR or in Group LR. According to this reasoning, the stronger preference in Group HL reflects the combined conditioning processes isolated in Groups HR and LR. In Experiment 1A, however, this relationship between groups was not observed; preference in Group HL was not appreciably larger than that observed in Group HR or Group LR. Procedural details that differed between experiments (e.g., amount of flavor solution presented in training, preliminary single-bottle testing in Experiment 1A) may account for this difference in results.

However, two observations in Experiment 1B suggest that the pairing of flavors with low deprivation is relatively more important than the association of flavors with high deprivation. First, conditioned flavor preference due to number of flavor-deprivation pairings was somewhat greater in Group L than in Group H. Second, a comparison of the differences in preference conditioned in Groups $H R$ and $H$ and those in Groups $L R$ and $L$ indicates that pairings with low deprivation may be relatively more influential than pairings with high deprivation. Recall that in Group HR the random-deprivation flavor was presented 5 times under both low- and highdeprivation conditions, whereas the low-deprivation flavor in Group $H$ was presented only 5 (rather than 10) times under high-deprivation conditions. The additional presentations of the random flavor under low-deprivation conditions in Group HR resulted in a conditioned preference for the low-deprivation flavor that was significantly greater in Group HR than in Group H. However, a parallel comparison of preference between groups LR and $L$ reveals that the 5 additional presentations of the random flavor under high deprivation in Group LR did not significantly reduce the conditioned preference in that group-Groups LR and $L$ did not differ in preference for their low-deprivation flavor. If, indeed, pairings with low deprivation are more influential than pairings with high deprivation, then the $\mathbf{R}$ condition is more like a low condition than a high one, and is not neutral. If this is so, then it is meaningful that a flavor associated only with low deprivation was preferred to one paired with both low and high deprivation (Group LR). However, greater preference for the randomly paired cue over the highdeprivation cue does not necessarily indicate that there was decreased preference of the high-deprivation cue. The 
latter preference may be due simply to the fact that the randomly paired cue was more like a low-deprivation cue than a neutral cue. Accordingly, in Experiment 2, we used a different "neutral" alternative.

\section{EXPERIMENT 2}

In Experiment 2, as in Experiment 1, we attempted to identify the value of flavors associated with low and high deprivation by comparing preferences for these flavors against some neutral alternative. In this case, subjects had one flavor paired exclusively with high deprivation and the other flavor paired exclusively with low deprivation; later in testing, the subjects were given simultaneous choice either between their previous high-deprivation flavor and the now unflavored base solution (Group $\mathrm{H}$ ) or between the previous low-deprivation flavor and the unflavored base solution (Group L). To be able to ascertain how the preference for a flavor might change as a result of conditioning, it was necessary to determine what the pretraining preferences for the two flavors and the unflavored base solution were. Consequently, a control group (Group CON), which was given only the unflavored base solution during training and then later a choice between the flavored and unflavored base solution, was included. By comparing the relative preferences for flavors in the two experimental groups against the flavor preference observed in the control group, we should be able to determine how preferences for flavors shifted as a result of pairing with either high or low deprivation. Greater preferences for flavors in the experimental groups would indicate conditioned preference. Weaker preferences for flavors in the experimental groups would indicate conditioned aversions.

\section{Method}

Subjects. The subjects were 36 experimentally naive male albino rats from the Holtzman Company; they were 81 days old upon arrival in our laboratory and $\mathbf{1 0 2}$ days old at the beginning of the experiment. Two subjects were eliminated due to illness, and their data were discarded.

Materials. The materials were the same as those used in Experiment 1 except that natural oil of cinnamon and oil of wintergreen (rather than imitation mixtures) were used to make the flavors.

Procedure. The training and testing procedures were identical to those in Experiment 1B except as indicated. Feeding on alternate days occurred at 5:00 p.m. and training and testing occurred at 8:30 a.m., resulting in 15 and $39 \mathrm{~h}$ between feeding and flavor presentation on low- and high-deprivation days, respectively. For subjects in Groups $H$ and $L$, one flavor of saccharin was given on high-deprivation days and the other flavor of saccharin was given on low-deprivation days ( $\mathrm{C}$ and $\mathrm{W}$ were counterbalanced). For subjects in Group CON, the control group, only unflavored saccharin solution was presented on both high- and low-deprivation days. All subjects were given $5 \mathrm{ml}$ of saccharin solution throughout training. Training solutions were presented on Days 11-20, 28-41, 56, and 57. On Day 21, because of experimenter error, subjects were not fed as scheduled; the following morning, the flavors usually associated with low deprivation were given under extra-high deprivation. On Days 22-27, no training solutions were presented while subjects readjusted to their normal deprivation schedule. On
Days 42-55, over a school break, all subjects were maintained on the same alternating feeding schedule.

On Days 58-64, all subjects were given two 10-min two-bottle choice tests between $40 \mathrm{ml}$ of one flavored solution and $40 \mathrm{ml}$ of a solution of exactly the same base solution, unflavored. In the experimental groups, half of the subjects were tested with their previous high-deprivation flavor (Group $\mathrm{H}$ ) and half were tested with their previous low-deprivation flavor (Group L). In the case of Group CON, the subjects had not previously been exposed to either flavor, so this preference test established the naturally occurring level of preference between a flavored base solution and the unflavored base solution. For half of the subjects in each of the three groups, the test flavor was $C$ and for the other half it was $W$. The tubes were removed after $10 \mathrm{~min}$, and there was a 10-15 min break before a second test was begun. Two tests were used to allow for refilling of tubes so that no rat would run out of solution. The position of flavored and unflavored tubes was counterbalanced among subgroups. This tube position alternated across days and between tests on a given day; amounts of residual solutions were recorded following each test. In no case was all $\mathbf{4 0 ~} \mathrm{ml}$ consumed.

\section{Results}

All solutions used in training were consumed.

The proportions of flavored solution consumed (milliliters flavored solution/total milliliters consumed) over the 12 days of testing were $.66, .51$, and .42 for Groups $L$, $\mathrm{H}$, and CON, respectively. Preference for the flavored solution was greater when it had previously been associated with low deprivation. Analysis of variance indicated that this difference between groups was significant $[F(2,28)=9.60, p<.001]$. Newman-Keuls tests showed that preference for the flavored solution was greater in Group L than in either Group H or Group CON and that the latter two groups did not differ significantly. This differential preference for the flavored solution in Group $L$ emerged in the first 2-day block of testing $[F(2,28)=7.07, p<.01]$ and remained consistent throughout. Overall, consumption of the flavored solution did increase over days, as indicated by a main effect of blocks $[F(5,140)=4.80, p<.001]$, but there was no blocks $\times$ group interaction $[F(10,140)=1.38$, $p>$.1]. An examination of the data revealed that, over test days, preference for the flavored solution over the unflavored base increased comparably in Groups $L$ and CON but that preference changed somewhat less in Group H.

Analysis of these data in terms of milliliters of flavored and unflavored solutions consumed yielded results parallel to those just described. Also, the results did not differ on the two tests within each test day or as a function of deprivation during test. These data are therefore not presented.

\section{Discussion}

The data obtained in this experiment suggest that the conditioning procedure under investigation results in a conditioned preference for the low-deprivation flavor rather than a conditioned aversion to the high-deprivation flavor. This is evidenced by the fact that preference for the low-deprivation flavor (Group L) is greater than the preference for the same flavor previously associated with 
high deprivation (Group H) or not previously exposed (Group CON).

It is possible that our measure of preconditioning flavor preference in the control group is low because of a neophobic reaction to the novel flavor. Given a choice between the novel flavored base solution and the same familiar but unflavored base solution, perhaps the rats chose the familiar unflavored solution over the unfamiliar flavored solution because of flavor neophobia. This factor could lead to a misinterpretation of the effects of preference conditioning in the experimental groups. This explanation of the data is not feasible, however, because there was no greater increase in preference for the flavored solution in Group CON than there was in Group L over days of testing. If subjects in Group CON were initially neophobic to flavors, then one would expect the relative flavor preference to change quite dramatically as the rats became familiar with the flavors.

It is possible that the apparent conditioned flavor preference in this experiment may have been influenced by the previous association of flavors with the saccharin base solution during training. Perhaps flavor consumption is influenced both by prior association with a specific deprivation condition and by association with the saccharin base solution in which it was presented. Such a combined conditioning effect could camouflage the actual effects of exposure to flavors under different deprivation conditions. To determine to what extent flavor preferences in this experiment were due to prior association of flavors with saccharin, in Experiment 3 we eliminated the saccharin from the base solution.

\section{EXPERIMENT 3}

In Experiment 3 our aim was to eliminate any conditioning of flavor preference due to prior pairing with saccharin. Procedures were modified so that during training experimental subjects consumed flavored water under high and low deprivation, and then during testing all subjects were given choice testing between flavored and unflavored water.

\section{Method}

Subjects. The subjects were 18 naive male rats from the Holtzman Company, 77 days old on arrival in our laboratory and 93 days old at the beginning of the experiment.

Materinls. No saccharin solution was used; flavors were presented in water throughout training and testing.

Procedure. The training and testing procedures were identical to those of the previous experiments except as modified to accommodate training with flavored water. On training days, two tubes, each containing $5 \mathrm{ml}$ of flavored (Groups $\mathrm{H}$ and $\mathrm{L}$ ) or unflavored water (Group CON), were placed on each cage front at 8:30 a.m. On high-deprivation days, these flavor tubes were removed prior to feeding at 5:00 p.m. and the water bottle was replaced on the cage front until 8:30 a.m. the next day. On low-deprivation days, the tubes were placed on the cage at 8:30 a.m. and not removed until the following morning. It was necessary to extend flavor exposure to get subjects to consume more of the flavored solutions. The two tubes were placed equally often in all combinations of the four tube positions employed later in testing.
On Days 31-38, all subjects were given 24-h choice tests between flavored and unflavored water. The flavor used in testing was the one associated with low deprivation in Group $L$ and with high deprivation in Group H. C and W were counterbalanced in all groups. Four tubes, each containing $\mathbf{4 0} \mathrm{ml}$ of water, two flavored and two unflavored, were placed on the cage front each morning at 8:00 a.m. and removed the following morning. Residual solutions were measured and recorded each day. This 24 h choice test was employed because the subjects did not readily consume the solutions when they were initially presented. To prevent the subjects from consuming all of either solution, thus potentially biasing the choice scores, two tubes of each solution were presented. Throughout training and testing, the positions of water and flavor tubes were varied according to a semirandom sequence, so that they were placed equally often in all possible combinations of four positions.

\section{Results}

Over all 8 days of testing, the subjects in Group L tended to consume more of the flavored water than did subjects in either Group H or Group CON. The proportion of flavored solution consumed was $.46, .38$, and $.35 \mathrm{ml}$ for Groups $\mathrm{L}, \mathrm{H}$, and $\mathrm{CON}$, respectively. However, analysis of variance indicated that these group differences were not significant $[F(2,12)=1.66$, $p>$.2].

Although group differences were not significant, the trend was the same as in the previous two experiments. Even though subjects in all groups consumed proportionately more of the unflavored water than of the flavored water, preference in the control group indicated that this was due to innate preferences for flavors rather than to any prior conditioning history of flavors. To the degree that prior pairing with deprivation had any effect (comparing experimental groups with the control group), it appears that prior consumption under low deprivation increased preference. These data are consistent with the assumption that presentation of flavors in a saccharin base solution results in an increased preference for the flavors as a result of the flavor-saccharin association. Again, there was no indication that anything aversive became associated with either the high- or low-deprivation flavors.

A number of factors differ between this experiment and previous experiments, and these may be responsible for our failure to observe group differences in preference for the flavored water. Because we did not know if our original observation of preference for the previous lowdeprivation flavor could be produced using this water training procedure, in Experiment 4 we attempted to replicate our original preference conditioning phenomenon using this water training procedure.

\section{EXPERIMENT 4}

\section{Method}

Subjects. The subjects were 24 experimentally naive rats from the Holtzman Company, 77 days old on arrival in our laboratory and 90 days old at the beginning of our experiment.

Materials. The materials used were the same as those in Experiment 3.

Procedure. The training procedure was identical to that used for the experimental groups in Experiment 3. Half of the subjects had 
$C$ paired with low deprivation and $W$ paired with high deprivation (Group CL); the other subjects had the opposite pairing (Group WL).

Testing involved 24-h choice testing between $\mathrm{C}$ - and W-flavored water. Four tubes, two of each flavor, were placed on the cage each morning and removed the following morning. All possible combinations of tube positions were employed in a semirandom sequence across days.

\section{Results}

Over all 8 days of testing, preference for flavors did not differ as a function of the deprivation level under which they were originally consumed. Although the proportion of W consumed was greater for Group WL than for Group CL (.42 and $.36 \mathrm{ml}$, respectively), indicating a somewhat greater preference for $W$ when it had previously been consumed under low deprivation, analysis of variance indicated that this difference was not significant $(F<1)$.

Analysis of variance of proportion scores on the first 2-day block of testing indicated that Group WL consumed relatively more $\mathbf{W}$-flavored water than did Group CL $[F(1,21)=4.34, p<.05]$. The proportion scores were .50 and .36 for Groups WL and CL, respectively. Analysis of variance on amounts consumed, however, showed that the group $\times$ flavor interaction was not significant $[F(1,21)=1.87, p>.10]$.

These data suggest that, for whatever reason, the water training procedure employed in Experiments 3 and 4 produces, at best, small and transitory preference for the previous low-deprivation flavor. This accounts for our failure to observe group differences in Experiment 3, and further suggests that this procedure is inappropriate for studying the kind of conditioned preferences observed when flavors are presented in sweet solutions.

\section{GENERAL DISCUSSION}

Taken as a whole, these experiments strongly suggest that the apparent preference for flavors previously consumed under low deprivation over flavors previously consumed under high deprivation is actually based on a conditioned preference for the low-deprivation flavor rather than a conditioned aversion to the high-deprivation flavor. Experiments 2-4 show no evidence that anything aversive becomes associated with the high-deprivation flavor. Rats that were exposed to flavors under low deprivation showed an increased preference for those flavors as compared with rats that were exposed to the same flavors under high deprivation or that had had no prior exposure to flavors. There was no evidence that flavor preference among control subjects, which had had no exposure to flavors prior to testing, was unduly suppressed due to neophobia; whatever small neophobic reaction there may have been could not explain the differential preferences between groups. Far from conditioning anything aversive, prior exposure to flavors in saccharin, under either high or low deprivation, as Experiment 3 indicates, results in a conditioned preference for flavors. This is somewhat more true for flavors consumed under low deprivation than for flavors consumed under high deprivation, although this difference was not found to be statistically significant.

Experiment 4 showed that when flavors are given in water there is no preference for the low-deprivation flavor. This is inconsistent with Davidson-Codjoe and Holman's (1982) hypothesis that the deprivation states themselves are being associated with the flavors, inasmuch as such an association should be made regardless of whether the flavors are given in water or in saccharin. This finding is also incompatible with Capaldi et al.'s (1983) hypothesis. If flavors taste better under low deprivation than under high deprivation, this should be equally true for flavors given in water and those given in saccharin. Perhaps when the taste of something is very bland, as water presumably is, a floor effect masks the difference in taste associated with deprivation. We have previously suggested that when the base solution is highly preferred (as with $8 \%$ sucrose), a ceiling effect masks the preference due to deprivation states. Thus, this view implies that only when flavors are in moderately preferred solutions will a flavor experienced previously under low deprivation be preferred to one previously experienced under high deprivation. Consistent with this hypothesis, base solutions of $.15 \%$ saccharin, $2 \%$ sucrose, and $20 \%$ sucrose have been used successfully to demonstrate a preference for the lowdeprivation flavor, whereas with the highly preferred $8 \%$ sucrose no preference is found for the low-deprivation flavor (Capaldi et al., 1983) and in Experiment 4 no preference was found with a water base solution.

In spite of the strong suggestive evidence for a preference conditioning mechanism, some may suggest that aversion conditioning mechanisms cannot be entirely ruled out. Since Experiment 3 demonstrated that preference for both high- and low-deprivation flavors is enhanced by association with the saccharin base solution, there is some small possibility that the effect of pairing flavors with deprivation conditions per se may result in some form of aversion conditioning that is manifest against a background of independently conditioned flavor preferences due to the pairing of flavors with saccharin. At least two of the observations reported seem to make such an account unlikely. First, when choice was given between flavored and unflavored saccharin (Experiment 2), not only did subjects show a stronger preference for the flavor when it had previously been consumed under low deprivation, but both experimental groups also consumed at least as much of the flavored as they did of the unflavored solutions (as indicated by proportion scores, flavored/flavored + unflavored $>.5$ ). This is hard to explain if one assumes that the effects of pairing with previous deprivation conditions, particularly high deprivation, results in a conditioned aversion to the flavor, and any previously conditioned preference for flavors is merely due to prior association with saccharin. It would only make sense if an innate preference for the flavored saccharin exceeded that for unflavored saccharin, and the control group in- 
dicated that that is not the case. Second, even when flavors were not previously associated with saccharin (Experiment 4), there was no indication that prior exposure to flavors under high deprivation reduced preference for flavors as compared with that of the control group. Thus, the present experiments provide no evidence that an aversion to the high-deprivation flavor is being conditioned. Instead, they suggest that it is a difference in preference between the flavor previously experienced under high deprivation and that consumed under low deprivation that is being conditioned.

\section{REFERENCES}

CAPAldi, E. D., M MYres, D. E. (1982). Taste preferences as a function of food deprivation during original taste exposure. Animal Learming \& Behavior, 10, 211-219.
Capaldi, E. D., Myers, D. E., Campbell, D. H., \& Sheffer, J. D. (1983). Conditioned flavor preferences based on hunger level during original flavor exposure. Arimal Leaming \& Behavior, 11, 107-115. Davidson-Codjoe, M., \& Holman, E. W. (1982). The effect of nonnutritive satiation on the learning of a flavor preference by rats. Animal Learning \& Behavior, 10, 220-222.

KURTZ, K. H., JARKA, R. G. (1968). Position preference based on differential food privation. Journal of Comparative \& Physiological Psychology, 66, 518-521.

Rescorla, R. A. (1967). Pavlovian conditioning and its proper control procedures. Psychological Review, 74, 71-80.

(Manuscript received November 25, 1985; revision accepted for publication September 12, 1986.) 